\title{
Non-existence of time-periodic solutions of the Dirac equation in a Reissner-Nordström black hole background
}

\author{
Felix Finster $^{\mathrm{a})}$ \\ Max Planck Institute for Mathematics in the Sciences, Inselstr. 22-26, 04103 Leipzig, \\ Germany \\ Joel Smoller ${ }^{\mathrm{b}}$ \\ Mathematics Department, The University of Michigan, Ann Arbor, Michigan 48109 \\ Shing-Tung Yauc) \\ Mathematics Department, Harvard University, Cambridge, Massachusetts 02138
}

(Received 12 October 1999; accepted for publication 25 October 1999)

It is shown analytically that the Dirac equation has no normalizable, time-periodic solutions in a Reissner-Nordström black hole background; in particular, there are no static solutions of the Dirac equation in such a background metric. The physical interpretation is that Dirac particles can either disappear into the black hole or escape to infinity, but they cannot stay on a periodic orbit around the black hole. (C) 2000 American Institute of Physics. [S0022-2488(00)01804-1]

\section{INTRODUCTION}

In recent years, there has been much interest in the gravitational collapse of matter to a black hole. Although both analytical ${ }^{1}$ and intensive numerical studies (see, e.g., Ref. 2) have given some understanding of how the event horizon and the singularity form, little is known about the asymptotic form of the black hole as $t \rightarrow \infty$. This is mainly due to the fact that standard numerical methods become unreliable after the solutions have formed singularities. Since all matter on a microscopic level is formed out of Dirac particles, it seems especially interesting to study the asymptotic collapse of a "cloud" of spin- $\frac{1}{2}$-particles. As a first step towards this goal, in this paper we study Dirac particles in a Reissner-Nordström background field.

We remark that considerable work has been done in the study of quantum mechanical wave equations in the presence of black holes. The papers which are most related are Refs. 3 and 4, where a massless Dirac particle is considered in a Schwarzschild metric background, and asymptotic completeness is shown for the scattering states near the event horizon and at infinity. However, the most physically interesting case of a massive Dirac particle near a charged black hole has not yet been considered. As we will see here, both the rest mass of the Dirac particle and the charge of the black hole lead to interesting physical effects and require new analytical tools.

In polar coordinates $(t, r, \vartheta, \varphi)$, the Reissner-Nordström metric has the form

$$
d s^{2}=\left(1-\frac{2 \rho}{r}+\frac{q^{2}}{r^{2}}\right) d t^{2}-\left(1-\frac{2 \rho}{r}+\frac{q^{2}}{r^{2}}\right)^{-1} d t^{2}-r^{2}\left(d \vartheta^{2}+\sin ^{2} \vartheta d \varphi^{2}\right),
$$

where $q$ is the charge of the black hole and $\rho$ its (ADM) mass. Furthermore, we have an external electromagnetic potential $A$ of the form $A=(-\phi, \overrightarrow{0})$ with the Coulomb potential

$$
\phi(r)=\frac{q}{r} .
$$

\footnotetext{
${ }^{\text {a)} E l e c t r o n i c ~ m a i l: ~ F e l i x . F i n s t e r @ m i s . m p g . d e ~}$

${ }^{b}$ Electronic mail: smoller@umich.edu

c)Electronic mail: yau@math.harvard.edu
} 
If $q<\rho$, the metric has two horizons; this is the so-called nonextreme case. If $q=\rho$, the metric has only one horizon at $r=\rho$; this extreme case describes a black hole at zero temperature; cf. Refs. $5-7$. For $q>\rho$, the metric does not describe a black hole, and thus this case will not be considered.

We describe the Dirac particles with "classical" wave functions (i.e., without second quantization). Both the gravitational and electric fields are coupled to the Dirac particles. We do not assume any spatial symmetry on the wave functions. Near a collapsing black hole, it seems reasonable that some of the Dirac particles could get into static or time-periodic states. Our main result is to show that this is not possible.

In the following we will restrict to time-periodic solutions, noting that static solutions are a special case. For classical point particles, the time-periodic solutions describe closed orbits of particles rotating around the black hole. Our goal is to investigate how this classical picture changes by the introduction of relativistic wave mechanics and spin. Since the phase of the Dirac wave function $\Psi$ is of no physical significance, we say that $\Psi$ is periodic with period $T$ if

$$
\Psi(t+T, r, \Theta, \varphi)=e^{-i \Omega T} \Psi(t, r, \vartheta, \varphi)
$$

for some real $\Omega$. Our main result in the nonextreme case is the following theorem:

Theorem I.1: In a nonextreme Reissner-Nordström black-hole background, there are no normalizable, periodic solutions of the Dirac equation.

In the extreme case, we prove a slightly weaker statement:

Theorem I.2: In an extreme Reissner-Nordström background, every normalizable, timeperiodic solution of the Dirac equation vanishes identically for $r>\rho$.

This surprising result shows that the classical picture breaks down completely; for Dirac particles, there are no periodic solutions. This means that Dirac particles which are attracted by a Reissner-Nordström black hole either "fall into" the singularity or escape to infinity, but they cannot stay on a periodic orbit around the black hole. The result can also be applied to the Dirac particles of the matter in the gravitational collapse; it then indicates that all the matter must eventually disappear in the black hole.

Basically, our result is a consequence of the Heisenberg uncertainty principle and of the particular form of the Dirac current. As a preparatory step, we analyze the behavior of the Dirac wave functions near the event horizon and we derive conditions which relate the wave function outside and inside the horizon. It is essential for our methods and results that the particles have spin. This shows that the spin is an important effect to be taken into account in the study of gravitational collapse.

In the remainder of this section, we give some basic formulas needed to describe Dirac particles in curved space-time (for a more detailed introduction to the classical Dirac theory in curved space-time see Ref. 8). In this paper, the Dirac equation is always of the form

$$
\left(i G^{j}(x) \frac{\partial}{\partial x^{j}}+\frac{i}{2}\left(\nabla_{j} G^{j}\right)(x)+e G^{j}(x) A_{j}(x)\right) \Psi(x)=m \Psi(x),
$$

where $m$ is the rest mass of the particle, $A=A_{j} d x^{j}$ is the electromagnetic potential, and $e$ is the electromagnetic coupling constant (see Refs. 9 and 10 for a derivation of this equation). The Dirac matrices $G^{j}(x)$ are real linear combinations of the usual $\gamma$-matrices. We work in the Dirac representation

$$
\gamma^{0}=\left(\begin{array}{cc}
1 & 0 \\
0 & -1
\end{array}\right), \quad \gamma^{i}=\left(\begin{array}{cc}
0 & \sigma^{i} \\
-\sigma^{i} & 0
\end{array}\right), \quad i=1,2,3
$$

where $\sigma^{i}$ denote the Pauli matrices. The Dirac matrices are related to the Lorentzian metric via the anticommutation relations

$$
g^{i k}(x)=\frac{1}{2}\left\{G^{j}(x), G^{k}(x)\right\} .
$$


The term $\nabla_{j} G^{j}$ in (I.4) is the divergence with respect to the Levi-Civita connection; it can be easily computed via the standard formula

$$
\nabla_{j} G^{j}=\frac{1}{\sqrt{|g|}} \partial_{j}\left(\sqrt{|g|} G^{j}\right)
$$

For the normalization of the wave functions, one takes a spacelike hypersurface $\mathcal{H}$ with normal vector field $\nu$ and considers the scalar product

$$
(\Psi \mid \Phi)=\int_{\mathcal{H}} \bar{\Psi} G^{j} \Phi \nu_{j} d \mu,
$$

where $\bar{\Psi}=\Psi^{*} \gamma^{0}$ is the adjoint spinor, and where $d \mu$ is the invariant measure on $\mathcal{H}$ induced by the Lorentzian metric. On solutions of the Dirac equation, we impose the normalization condition

$$
(\Psi \mid \Psi)=1 \text {. }
$$

Current conservation

$$
\nabla_{j} \bar{\Psi} G^{j} \Psi=0
$$

implies that this normalization condition remains unchanged if the hypersurface $\mathcal{H}$ is continuously deformed.

\section{THE DIRAC OPERATOR IN A SCHWARZSCHILD BACKGROUND}

We begin by analyzing the Dirac operator in a Schwarzschild background metric. Our aim is to analyze the behavior of the spinors near the event horizon. To do this, we must consider the Dirac equation in different coordinate systems.

\section{A. The Dirac operator in polar coordinates}

In polar coordinates $(t, r, \vartheta, \varphi)$, the Schwarzschild metric is

$$
d s^{2}=\left(1-\frac{2 \rho}{r}\right) d t^{2}-\left(1-\frac{2 \rho}{r}\right)^{-1} d r^{2}-r^{2}\left(d \vartheta^{2}+\sin ^{2} \vartheta d \varphi^{2}\right)
$$

where $\rho$ is the (ADM) mass. The metric has an event horizon at $r=2 \rho$. In order to derive the Dirac operator, we first choose Dirac matrices $G^{j}(x)$ satisfying the anticommutation relations (I.6). The Dirac operator is then obtained by calculating the divergence (I.7) and substituting into (I.4). [We point out that the choice of the Dirac matrices is not canonical; there are different real linear combinations of the $\gamma$-matrices which satisfy (I.6) However, the Dirac operators corresponding to different choices of the Dirac matrices are equivalent in the sense that they can be obtained from each other by a suitable local transformation of the spinors (see, e.g., Ref. 8). For this reason, we can simply choose the $G^{j}$ in the way which is most convenient to us.]

Outside the horizon, we can satisfy the anticommutation relations (I.6) by choosing the Dirac matrices in the form

$$
G^{t}=\frac{1}{S} \gamma^{t}, \quad G^{r}=S \gamma^{r}, \quad G^{\vartheta}=\gamma^{\vartheta}, \quad G^{\varphi}=\gamma^{\varphi} \quad(r>2 \rho)
$$

with 


$$
S(r)=\left|1-\frac{2 \rho}{r}\right|^{1 / 2}
$$

where $\gamma^{t}, \gamma^{r}, \gamma^{\vartheta}$, and $\gamma^{\varphi}$ are the " $\gamma$-matrices in polar coordinates"

$$
\begin{gathered}
\gamma^{t}=\gamma^{0}, \\
\gamma^{r}=\gamma^{3} \cos \vartheta+\gamma^{1} \sin \vartheta \cos \varphi+\gamma^{2} \sin \vartheta \sin \varphi, \\
\gamma^{\vartheta}=\frac{1}{r}\left(-\gamma^{3} \sin \vartheta+\gamma^{1} \cos \vartheta \cos \varphi+\gamma^{2} \cos \vartheta \sin \varphi\right), \\
\gamma^{\varphi}=\frac{1}{r \sin \vartheta}\left(-\gamma^{1} \sin \varphi+\gamma^{2} \cos \varphi\right) .
\end{gathered}
$$

The divergence of the Dirac matrices is computed to be

$$
\nabla_{j} G^{j}=\left(S^{\prime}+\frac{2}{r}(S-1)\right) \gamma^{r}
$$

Substituting into (I.4), we obtain for the Dirac operator, $G_{\text {out }}$, in the region $r>2 \rho$

$$
G_{\text {out }}=\frac{i}{S} \gamma^{t} \frac{\partial}{\partial t}+\gamma^{r}\left(i S \frac{\partial}{\partial r}+\frac{i}{r}(S-1)+\frac{i}{2} S^{\prime}\right)+i \gamma^{\vartheta} \frac{\partial}{\partial \vartheta}+i \gamma^{\varphi} \frac{\partial}{\partial \varphi} .
$$

For the normalization, we integrate over the hypersurface $t=$ const; i.e.,

$$
(\Psi \mid \Psi)_{\text {out }}^{t}:=\int_{\mathbb{R}^{3} \backslash B_{2 \rho}}\left(\bar{\Psi} \gamma^{t} \Psi\right)(t, \vec{x}) S^{-1} d^{3} x,
$$

where $B_{2 \rho}$ denotes the ball of radius $2 \rho$ around the origin. This normalization integral is problematic near the event horizon, as will be discussed in detail later. Inside the horizon, we must take into account that the radial direction $r$ is timelike, whereas $t$ is a space coordinate. So, in this region, to obtain the Dirac matrices, we reverse the roles of the matrices $\gamma^{t}$ and $\gamma^{r}$,

$$
G^{t}=\frac{1}{S} \gamma^{r}, \quad G^{r}=-S \gamma^{t}, \quad G^{\vartheta}=\gamma^{\vartheta}, \quad G^{\varphi}=\gamma^{\varphi} \quad(r<2 \rho) .
$$

The divergence of the Dirac matrices now has the form

$$
\nabla_{j} G^{j}=-\frac{2}{r} \gamma^{r}-\left(S^{\prime}+\frac{2}{r} S\right) \gamma^{t}
$$

Thus the Dirac operator, $G_{\text {in }}$, in the region $r<2 \rho$ is given by

$$
G_{\text {in }}=\gamma^{r}\left(\frac{i}{S} \frac{\partial}{\partial t}-\frac{i}{r}\right)-\gamma^{t}\left(i S \frac{\partial}{\partial r}+\frac{i}{r} S+\frac{i}{2} S^{\prime}\right)+i \gamma^{\vartheta} \frac{\partial}{\partial \vartheta}+i \gamma^{\varphi} \frac{\partial}{\partial \varphi} .
$$

According to (I.8), the naive extension of the normalization integral (II.6) to the interior of the horizon is

$$
(\Psi \mid \Psi)_{\mathrm{in}}^{t}:=\int_{B_{2 \rho}}\left(\bar{\Psi} \gamma^{r} \Psi\right)(t, \vec{x}) S^{-1} d^{3} x ;
$$


this will also be discussed in detail later.

Notice that as a particular freedom in the choice of the Dirac matrices, the signs in (II.1) and (II.7) are arbitrary. As remarked above, this arbitrariness can be compensated by a suitable local transformation of the spinors. However, this transformation of the spinors may change the sign of the scalar product (I.8). This is a subtle point which needs some explanation. Assume that we consider the spacelike hypersurface outside the horizon

$$
\mathcal{H}_{1}=\{t=\text { const }, r>2 \rho\} .
$$

Its normal vector field $\nu$ is only determined up to a sign. Depending on whether we choose for $\nu$ the future- or past-directed normals, the corresponding scalar product (I.8) will (for a fixed choice of the Dirac matrices $G^{j}$ ) be either positive or negative (semi-)definite. However, the overall sign of the scalar product is of no physical relevance; e.g., we could just redefine (I.8) by inserting a minus sign. In order to fix the sign convention, we will in the following always assume that the scalar product (I.8) is positive for the future-directed normal vector field [this convention is consistent with our choices (II.1) and (II.6)]. The situation becomes more interesting if we also look at the region inside the horizon. For this, we consider the "cylindric" spacelike hypersurface

$$
\mathcal{H}_{2}=\left\{r=r_{0}, t_{0} \leqslant t \leqslant t_{1}\right\}
$$

for some fixed $r_{0}<2 \rho$ and $t_{0}<t_{1}$. A short computation shows that, for our choice of the Dirac matrices (II.7), the scalar product (I.8) corresponding to $\mathcal{H}_{2}$ is positive if we choose for $\nu$ the inner normal (pointing towards the singularity at $r=0$ ). According to our sign convention, this means that the inward radial direction points to the future. Thus the particles "fall into" the singularity as time progresses, and we have a black hole. On the other hand, we could have chosen the Dirac matrices such that the scalar product corresponding to $\mathcal{H}_{2}$ is positive for the outer normal [e.g., by changing the sign of $G^{r}$ in (II.7)]. In this case, increasing $r$ would correspond to going forward in time, and we would have a white hole. Notice that this argument is consistent with time reversals. Namely, the replacement $t \rightarrow-t$ forces us to change the sign of the scalar product (I.8) [in order that (I.8) is still positive for $\mathcal{H}=\mathcal{H}_{1}$ and future-directed normals]. As a consequence, the scalar product corresponding to $\mathcal{H}_{2}$ changes sign. This means that black holes become white holes and vice versa. We conclude that the Dirac operators $G_{\text {out }}$ and $G_{\text {in }}$ distinguish between a black and a white hole. This is a peculiar effect of the Dirac operator. It is quite different from, e.g., the wave operator describing scalar fields (the Klein-Gordon operator), which does not determine the direction of time inside the horizon.

Our description of the spinors in polar coordinates is not quite satisfactory. First of all, the normalization integral inside the horizon, (II.9), is not definite. This is a consequence of the fact that the $t$-variable is spacelike inside the horizon. From the mathematical point of view, this is no problem; it seems tempting to just integrate across the horizon by adding (II.6) and (II.9). On the other hand, it is a conceptual difficulty that the integrand in (II.9) is not positive and therefore does not have the interpretation as a probability density. Furthermore, the Dirac equations corresponding to $G_{\text {out }}$ and $G_{\text {in }}$ separately describe the wave functions outside and inside the horizon. But it is not clear how to match the wave functions on the horizon. For a better understanding of these issues, it is useful to remove the singularity of the metric on the horizon by transforming to Kruskal coordinates.

\section{B. Kruskal coordinates}

According to Ref. 11, we introduce Kruskal coordinates $u$ and $v$ by

$$
u= \begin{cases}\sqrt{\frac{r}{2 \rho}-1} e^{r / 4 \rho} \cosh \left(\frac{t}{4 \rho}\right) & \text { for } r>2 \rho, \\ \sqrt{1-\frac{r}{2 \rho}} e^{r / 4 \rho} \sinh \left(\frac{t}{4 \rho}\right) & \text { for } r<2 \rho,\end{cases}
$$




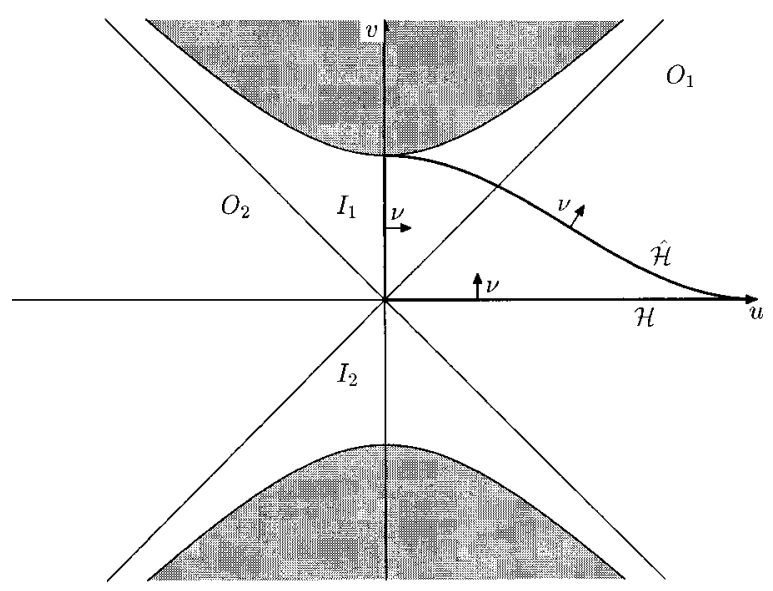

FIG. 1. Kruskal coordinates.

$$
u= \begin{cases}\sqrt{\frac{r}{2 \rho}-1} e^{r / 4 \rho} \sinh \left(\frac{t}{4 \rho}\right) & \text { for } r>2 \rho, \\ \sqrt{1-\frac{r}{2 \rho}} e^{r / 4 \rho} \cosh \left(\frac{t}{4 \rho}\right) & \text { for } r<2 \rho,\end{cases}
$$

The regions $r>2 \rho$ outside and $r<2 \rho$ inside the horizon are mapped into

$$
O_{1}=\{u>0,|v|<u\}
$$

and

$$
I_{1}=\left\{v>0,|u|<v, v^{2}-u^{2}<1\right\}
$$

respectively (see Fig. 1). The horizon $r=2 \rho$ corresponds to the origin $u=0=v$, and the lines $v$ $= \pm u$ are reached in the limit $t \rightarrow \pm \infty$. Finally, the singularity at $r=0$ corresponds to the hyperbola $v^{2}-u^{2}=1, v>0$.

In Kruskal coordinates $(v, u, \vartheta, \varphi)$, the Schwarzschild metric takes the form

$$
d s^{2}=f^{-2}\left(d v^{2}-d u^{2}\right)-r^{2}\left(d \vartheta^{2}+\sin ^{2} \vartheta d \varphi^{2}\right)
$$

with

$$
f^{-2}=\frac{32 \rho^{3}}{r} e^{-r / 2 \rho}
$$

This metric is regular except at the singularity $v^{2}-u^{2}=1$; it can be extended to the entire region $v^{2}-u^{2}<1$.

Since the metric is regular at the origin, we can smoothly extend the Dirac operator across the horizon. To do this, we simply view $v$ and $u$ as the time and space variables, respectively. We choose for the Dirac matrices

$$
G^{v}=f \gamma^{t}, \quad G^{u}=f \gamma^{r}, \quad G^{\vartheta}=\gamma^{\vartheta}, \quad G^{\varphi}=\gamma^{\varphi} .
$$

A straightforward computation yields for the Dirac operator 


$$
G=\gamma^{t}\left(f i \frac{\partial}{\partial v}+\frac{i}{r} f\left(\partial_{v} r\right)-\frac{i}{2} \partial_{v} f\right)+\gamma^{r}\left(f i \frac{\partial}{\partial u}+\frac{i}{r}\left(f\left(\partial_{u} r\right)-1\right)-\frac{i}{2} \partial_{u} f\right)+i \gamma^{\vartheta} \partial_{\vartheta}+i \gamma^{\varphi} \partial_{\varphi}
$$

The normalization integrals (II.6) and (II.9) on the surface $t=0$ correspond in Kruskal coordinates to the integral (I.8) with

$$
\mathcal{H}=\{u=0,0 \leqslant v \leqslant 1\} \cup\{v=0, u>0\} .
$$

We choose the normal $\nu$ as in Fig. 1. Using the current conservation (I.9), one can continuously deform the hypersurface $\mathcal{H}$ without changing the value of the normalization integral. In particular, we can avoid integrating across the horizon by choosing the hypersurface $\hat{\mathcal{H}}$ in Fig. 1 . This is a major advantage of Kruskal coordinates; it gives a physically reasonable positive normalization integral even inside the event horizon. However, this method must be done with care when the considered solution of the Dirac equation has singularities near the origin. Unfortunately, our time-periodic solutions of the Dirac equation will, after transforming to Kruskal coordinates, in general be highly singular at the origin. Therefore, the deformation of the hypersurface as in Fig. 1 would be problematic, and we will not use this method. In order to avoid any difficulties of the normalization integral near the horizon, we shall only consider the normalization integral outside and away from the event horizon.

\section{Transformation of the Dirac operator}

We now consider how the Dirac operator (II.5) and (II.8) in polar coordinates transforms into the Dirac operator (II.14) in Kruskal coordinates. This transformation consists of transforming both the space-time coordinates and the spinors. For clarity, we perform these transformations in two separate steps. Under the transformation of the space-time coordinates, the partial derivatives transform as

$$
\begin{gathered}
\frac{\partial}{\partial t}=\frac{\partial v}{\partial t} \frac{\partial}{\partial v}+\frac{\partial u}{\partial t} \frac{\partial}{\partial u}=\frac{1}{4 \rho}\left(u \frac{\partial}{\partial v}+v \frac{\partial}{\partial u}\right), \\
\frac{\partial}{\partial r}=\frac{\partial v}{\partial r} \frac{\partial}{\partial v}+\frac{\partial u}{\partial r} \frac{\partial}{\partial u}=\left\{\begin{array}{cc}
\frac{1}{4 \rho S^{2}}\left(v \frac{\partial}{\partial v}+u \frac{\partial}{\partial u}\right), & \text { for } r>2 \rho, \\
-\frac{1}{4 \rho S^{2}}\left(v \frac{\partial}{\partial v}+u \frac{\partial}{\partial u}\right), & \text { for } r<2 \rho
\end{array}\right.
\end{gathered}
$$

Substituting into (II.5) and (II.8) gives for the Dirac operators $G_{\text {out }}$ and $G_{\text {in }}$ in Kruskal coordinates

$$
\begin{aligned}
G_{\mathrm{out}}= & \frac{i}{4 \rho S}\left(u \gamma^{t}+v \gamma^{r}\right) \frac{\partial}{\partial v}+\frac{i}{4 \rho S}\left(v \gamma^{t}+u \gamma^{r}\right) \frac{\partial}{\partial u} \\
& +\left(\frac{i}{r}(S-1)+\frac{i}{2} S^{\prime}\right) \gamma^{r}+i \gamma^{\vartheta} \frac{\partial}{\partial \vartheta}+i \gamma^{\varphi} \frac{\partial}{\partial \varphi} \\
G_{\mathrm{in}}= & \frac{i}{4 \rho S}\left(v \gamma^{t}+u \gamma^{r}\right) \frac{\partial}{\partial v}+\frac{i}{4 \rho S}\left(u \gamma^{t}+v \gamma^{r}\right) \frac{\partial}{\partial u} \\
& -\left(\frac{i}{r} S+\frac{i}{2} S^{\prime}\right) \gamma^{t}-\frac{i}{r} \gamma^{r}+i \gamma^{\vartheta} \frac{\partial}{\partial \vartheta}+i \gamma^{\varphi} \frac{\partial}{\partial \varphi}
\end{aligned}
$$

These Dirac operators do not coincide with (II.14), and we must therefore perform a further transformation; namely a transformation of the spinors. Under general coordinate transformations, the wave functions transform according to 


$$
\Psi(x) \rightarrow U(x) \Psi(x)
$$

in the case considered here, $U$ is the time-dependent $(4 \times 4)$ matrix

$$
U(t)=\cosh \left(\frac{t}{8 \rho}\right) 1+\sinh \left(\frac{t}{8 \rho}\right) \gamma^{t} \gamma^{r}
$$

Under this transformation, the Dirac operators $G_{\text {out/in }}$ transform as

$$
G_{\text {out } / \text { in }} \rightarrow U G_{\text {out } / \text { in }} U^{-1} \text {. }
$$

This gives the Dirac operator (II.14) in Kruskal coordinates,

$$
G=U G_{\text {out }} U^{-1}=U G_{\text {in }} U^{-1}
$$

and this can be verified as follows: Under the transformation (II.19), the Dirac matrices behave like

$$
G^{j}(x) \rightarrow U(x) G^{j}(x) U(x)^{-1}
$$

Using the form of the Dirac matrices in (II.15) and (II.16) and the explicit formula (II.18), a short calculation shows that the Dirac matrices of the operators $U G_{\text {out } / \text { in }} U^{-1}$ coincide with the Dirac matrices $f \gamma^{t}, f \gamma^{r}, \gamma^{\vartheta}$, and $\gamma^{\varphi}$ in (II.14). According to (I.4), the Dirac operator in the gravitational field is formed from the Dirac matrices and their covariant derivatives; it is thus completely determined by the Dirac matrices. Therefore, the operators $U G_{\text {out/in }} U^{-1}$ must coincide with $G$. (One can also verify explicitly that the zeroth-order terms of the operators $U G_{\text {out } / \text { in }} U^{-1}$ and $G$ are equal. This is a longer computation, however.) We conclude that the Dirac operators $G_{\text {out }}$ and $G_{\text {in }}$ can be identified with the Dirac operator $G$ in the region $O_{1} \cup I_{1}=\left\{u+v>0, v^{2}-u^{2}<1\right\}$.

We remark that it is not possible to map the interior of the horizon into the region

$$
I_{2}=\left\{v<0,|u|<-v, v^{2}-u^{2}<1\right\}
$$

and still match the Dirac operator $G_{\text {in }}$ with $G$, because this would contradict the fact discussed in the previous section that the Dirac operator distinguishes between black and white holes.

Finally, we note that the transformation (II.17) and (II.19) of the spinors can be viewed as a local U $(2,2)$ gauge transformation; see Ref. 8.

\section{Matching of the spinors on the horizon}

We now come to the question of how the wave functions inside and outside the horizon are related to each other. For this, we analyze the behavior of solutions of the Dirac equation at the origin in Kruskal coordinates. After transforming back to polar coordinates, this will give matching conditions for the wave functions on the event horizon. The physical situation which we have in mind is a Dirac particle attracted by a Schwarzschild black hole. It suffices to do the matching for static solutions (and not time-periodic solutions), since in Sec. IV, we reduce the problem to static solutions.

Let $\Psi$ be a static wave function, i.e., in polar coordinates

$$
\Psi(t, r, \vartheta, \varphi)=e^{-i \omega t} \Psi(r, \vartheta, \varphi)
$$

We assume that $\Psi(r, \vartheta, \varphi)$ is a smooth function both inside and outside the horizon, i.e., in the regions $r<2 \rho$ and $r>2 \rho$; this will be justified later by a separation of variables technique. Furthermore, we assume that $\Psi$ is a solution of the Dirac equations $\left(G_{\text {in }}-m\right) \Psi=0$ and $\left(G_{\text {out }}\right.$ $-m) \Psi=0$, respectively. According to the transformation rules (II.17) and (II.18), the wave function $\Psi$ in Kruskal coordinates takes the form 


$$
\Psi(u, v, \vartheta, \varphi)=U(t) e^{-i \omega t} \Psi(r, \vartheta, \varphi)
$$

where $r$ and $t$ are given implicitly in terms of $u$ and $v$ by

$$
\begin{gathered}
\left(\frac{r}{2 \rho}-1\right) e^{r / 2 \rho}=u^{2}-v^{2}, \\
\tanh \left(\frac{t}{4 \rho}\right)= \begin{cases}\frac{v}{u} & \text { for } r>2 \rho, \\
\frac{u}{v} & \text { for } r<2 \rho .\end{cases}
\end{gathered}
$$

With this procedure, $\Psi$ is only defined in $O_{1} \cup I_{1}$, the upper right half of the Kruskal domain. It solves the Dirac equation

$$
(G-m) \Psi=0
$$

in the open set $O_{1} \cup I_{1}$. If $\Psi$ is to be a physically reasonable solution of the Dirac equation, it must be possible to extend it to the entire region $v^{2}-u^{2}<1$ between the two hyperbolas. If this extended wave function was not zero in the region $u+v<0$, our system would be connected to a white hole or to another universe (through a worm hole), and the Dirac particle would have a certain probability to be in these extensions of space-time. Since we are only interested in black holes, this is not the situation we want to consider. Therefore, we demand that the extension of $\Psi$ must vanish identically in the half-plane $u+v<0$. We conclude that in Kruskal coordinates, we must analyze a solution $\Psi$ of the Dirac equation (II.23) of the form

$$
\Psi(u, v, \vartheta, \varphi)=\left\{\begin{array}{l}
U(t) e^{-i \omega t} \Psi(r, \vartheta, \varphi) \quad \text { for } u+v>0, u \neq v, \\
0 \quad \text { for } u+v<0 .
\end{array}\right.
$$

This wave function may be singular on the lines $u= \pm v$; in this case, $\Psi$ must solve the Dirac equation in a generalized weak sense.

For the calculation of the weak derivatives of $\Psi$, we rewrite the wave function in the form

$$
\Psi=\Theta(u+v) \Theta(u-v) \Psi_{O}+\Theta(v+u) \Theta(v-u) \Psi_{I},
$$

where $\Psi_{O}=\Psi_{\mid O_{1}}$ and $\Psi_{I}=\Psi_{\mid I_{1}}$ are the components of $\Psi$ outside, resp. inside, the horizon [ $\Theta$ denotes the Heaviside function $\Theta(x)=1$ for $x \geqslant 0$ and $\Theta(x)=0$ otherwise]. Since $\Psi$ satisfies the Dirac equation in $O_{1} \cup I_{1}$, we need only consider the singular contributions on the lines $u$ $= \pm v$. A formal calculation gives

$$
\begin{gathered}
0=(G-m) \Psi= \\
\quad f i\left(\gamma^{t}+\gamma^{r}\right) \delta(u+v)\left(\Theta(u-v) \Psi_{O}+\Theta(v-u) \Psi_{I}\right) \\
-f i\left(\gamma^{t}-\gamma^{r}\right) \delta(u-v) \Theta(u+v)\left(\Psi_{O}-\Psi_{I}\right) .
\end{gathered}
$$

If $\Psi_{O}$ and $\Psi_{I}$ were smooth up to the boundary of $O_{1}$, resp. $I_{1}$, this equation would be well defined in the distributional sense. In general, however, $\Psi_{O}$ and $\Psi_{I}$ might be singular in the limit $u \rightarrow \pm v$. In order to treat this general case, we multiply (II.25) and (II.26) with test functions $\eta(u, v)$ which, as $u \rightarrow \pm v$, decay so fast that the integral over the resulting expression is well defined. Since the matrices $\left(\gamma^{t}+\gamma^{r}\right)$ and $\left(\gamma^{t}-\gamma^{r}\right)$ are linearly independent, we get the two conditions

$$
\int_{\mathbb{R}^{2}} \eta f \delta(u+v)\left(\gamma^{t}+\gamma^{r}\right)\left(\Theta(u-v) \Psi_{O}+\Theta(v-u) \Psi_{I}\right) d u d v=0
$$




$$
\int_{\mathbb{R}^{2}} \eta f \delta(u-v)\left(\gamma^{t}-\gamma^{r}\right) \Theta(u+v)\left(\Psi_{O}-\Psi_{I}\right) d u d v=0 .
$$

In (II.27), we compensate the possible divergence of $\Psi$ for $u \rightarrow-v$ by choosing $\eta$ in the region $O_{1} \cup I_{1}$ to be of the form $\eta_{\mid O_{1} \cup I_{1}}=\left(1+\left|\left(\gamma^{t}+\gamma^{r}\right) \Psi\right|\right)^{-1} g$ with a smooth function $g$. Then the integrand in (II.27) is of the form $\delta(u+v) \times g \times$ (bounded function), and the integral makes sense. Since $g$ is arbitrary, we conclude that the integrand on the line $u=-v$ must vanish, which implies that

$$
\lim _{u \rightarrow-v}\left(\gamma^{t}+\gamma^{r}\right) \Psi(u, v, \vartheta, \varphi)=0 .
$$

In (II.28), we can proceed similarly; namely, if $\Psi$ is singular on the line $u=v$, we compensate the divergence of the integrand in (II.28) by choosing $\eta$ to have an appropriately fast decay near the line $u=v$. One must keep in mind, however, that $\eta$ cannot be chosen independently in $O_{1}$ and $I_{1}$, because the smoothness of $\eta$ on the line $u=v$ may impose restrictions on $\eta$. For example, if $\Psi_{O}$ and $\Psi_{I}$ have poles near $u=v$,

$$
\begin{gathered}
\Psi_{I}(u, u+\varepsilon, \vartheta, \varphi)=c_{1}(u, \vartheta, \varphi) \varepsilon^{-p}+\cdots, \\
\Psi_{O}(u, v-\varepsilon, \vartheta, \varphi)=c_{2}(u, \vartheta, \varphi) \varepsilon^{-q}+\cdots, \quad(\varepsilon>0),
\end{gathered}
$$

then we must choose $\eta$ in the form

$$
\eta(u, u+\varepsilon, \vartheta, \varphi)=c_{3}(u, \vartheta, \varphi) \varepsilon^{\max (p, q)}+\cdots \quad(\varepsilon>0 \text { or } \varepsilon<0) .
$$

Thus the asymptotic behavior of $\eta$ near $u=v$ in $O_{1}$ and $I_{1}$ must be the same. In the integral (II.28), this means that the leading order singularities of $\Psi_{O}$ and $\Psi_{I}$ may cancel each other for any choice of $\eta$. Therefore, the condition for the leading order singularity takes the form

$$
\begin{aligned}
& \quad\left(\gamma^{t}-\gamma^{r}\right)(\Psi(u, u+\varepsilon, \vartheta, \varphi)-\Psi(u, u-\varepsilon, \vartheta, \varphi)) \\
& \quad=o\left(1+\left|\left(\gamma^{t}-\gamma^{r}\right) \Psi(u, u+\varepsilon, \vartheta, \varphi)\right|\right) \quad \text { as } \varepsilon \rightarrow 0 .
\end{aligned}
$$

If the singularity of $\Psi$ on the line $u=v$ is worse than polynomial or of different form, there may be no obstructions for the choice of $\eta$ in $O_{1}$ and $I_{1}$. In this case, (II.30) will still be a necessary condition. It will no longer be the strongest possible condition, but this is irrelevant for our purposes. For simplicity, we will use (II.30) in the general case.

Next we evaluate the conditions (II.29) and (II.30) for our wave function $\Psi(u, v, \vartheta, \varphi)$ in (II.24). Using (II.18), we have in $O_{1} \cup I_{1}$

$$
\begin{gathered}
\left(\gamma^{t}+\gamma^{r}\right) \Psi(u, v, \vartheta, \varphi)=\left(\gamma^{t}+\gamma^{r}\right) e^{t / 8 \pi} e^{-i \omega t} \Psi(r, \vartheta, \varphi), \\
\left(\gamma^{t}-\gamma^{r}\right) \Psi(u, v, \vartheta, \varphi)=\left(\gamma^{t}-\gamma^{r}\right) e^{-t / 8 \pi} e^{-i \omega t} \Psi(r, \vartheta, \varphi) .
\end{gathered}
$$

The explicit formulas (II.12) and (II.13) enable us to write the time exponential in terms of $u$ and $v$ as

$$
e^{ \pm t / 8 \rho}=\left|\frac{u+v}{u-v}\right|^{ \pm 1 / 4}
$$

Using the relation (II.21) between $r, u$, and $v$, the condition (II.29) in polar coordinates takes the form

$$
\lim _{\varepsilon \rightarrow 0}\left(\gamma^{t}+\gamma^{r}\right)|\varepsilon|^{1 / 4} \Psi(t, 2 \rho+\varepsilon, \vartheta, \varphi)=0 .
$$


Similarly, condition (II.30) can be written in polar coordinates as

$$
\begin{aligned}
& \left(\gamma^{t}-\gamma^{r}\right)|\varepsilon|^{1 / 4}(\Psi(2 \rho+\varepsilon, \vartheta, \varphi)-\Psi(2 \rho-\varepsilon, \vartheta, \varphi)) \\
& \quad=o\left(1+|\varepsilon|^{1 / 4}\left|\left(\gamma^{t}-\gamma^{r}\right) \Psi(2 \rho+\varepsilon, \vartheta, \varphi)\right|\right) \quad \text { as } \varepsilon \rightarrow 0
\end{aligned}
$$

In order to simplify this formula, we consider the decomposition of $\Psi$ in the form

$$
|\varepsilon|^{1 / 4} \Psi=\frac{1}{2} \gamma^{t}\left(\left(\gamma^{t}+\gamma^{r}\right)|\varepsilon|^{1 / 4} \Psi+\left(\gamma^{t}-\gamma^{r}\right)|\varepsilon|^{1 / 4} \Psi\right)
$$

According to condition (II.31), the first summand in the bracket in (II.33) vanishes on the horizon $r=2 \rho$. Since the matrix $\gamma^{t}$ is invertible, we conclude that $\Psi$ and $\left(\gamma^{t}-\gamma^{r}\right) \Psi$ are of the same order on the horizon. Thus we can take out the matrices $\left(\gamma^{t}-\gamma^{r}\right)$ in (II.32) and finally obtain the equivalent condition

$$
|\varepsilon|^{1 / 4}(\Psi(t, 2 \rho+\varepsilon, \vartheta, \varphi)-\Psi(t, 2 \rho-\varepsilon, \vartheta, \varphi))=o\left(1+|\varepsilon|^{1 / 4} \Psi(t, 2 \rho+\varepsilon, \vartheta, \varphi)\right)
$$

as $\varepsilon \rightarrow 0$. The relations (II.31) and (II.34) are our matching conditions.

We briefly explain what these matching conditions mean, without being mathematically precise. First of all, we point out that the matrix $\left(\gamma^{t}+\gamma^{r}\right)$ in the first matching condition (II.31) is not invertible. Therefore, (II.31) does not imply that $|\varepsilon|^{1 / 4} \Psi(2 \rho+\varepsilon, \vartheta, \varphi)$ goes to zero in the limit $\varepsilon \rightarrow 0$; in general, this limit need not even exist. Although the matching conditions have a quite special form, they can be understood intuitively if one considers the Dirac current in polar coordinates. We first look at the total normalization integral (II.6) +(II.9):

$$
(\Psi \mid \Psi)_{\text {out }}+(\Psi \mid \Psi)_{\text {in }}=\int_{B_{2 \rho}} \bar{\Psi}\left(\gamma^{r}+\gamma^{t}\right) \Psi d \mu-\int_{B_{2 \rho}} \bar{\Psi} \gamma^{t} \Psi d \mu+\int_{\mathbb{R}^{3} B_{2 \rho}} \bar{\Psi} \gamma^{t} \Psi d \mu .
$$

The condition (II.31) ensures that the integral of the first summand is small near the horizon. Using the matching condition (II.34), one sees that the integrals in the second and last summands behave similarly near the horizon. Because of the opposite sign of the second and third summands, this tends to make the normalization integral finite even if $\Psi$ is singular on the horizon (if the current had a pole, for example, one could define the normalization integral as a principal value). Thus our matching conditions "regularize" the normalization integral across the horizon. It is also interesting to look at the current in radial direction. For this, we consider the normalization integral through the hypersurface $\mathcal{H}_{2}$, (II.11). For the outer normal $\nu$, this gives inside the horizon

$$
(\Psi \mid \Psi)_{\mathcal{H}_{2}}=-\int_{\mathcal{H}_{2}} \bar{\Psi} \gamma^{t} \Psi d \mu \quad(r<2 \rho)
$$

For $r>2 \rho$, on the other hand, we get the expression

$$
(\Psi \mid \Psi)_{\mathcal{H}_{2}}=\int_{\mathcal{H}_{2}} \bar{\Psi} \gamma^{4} \Psi d \mu=\int_{\mathcal{H}_{2}} \bar{\Psi}\left(\gamma^{r}+\gamma^{t}\right) \Psi d \mu-\int_{\mathcal{H}_{2}} \bar{\Psi} \gamma^{t} \Psi d \mu \quad(r>\rho)
$$

According to (II.31), the first integral in (II.36) is small near the horizon $r=2 \rho$. The matching condition (II.34) gives that the second summand in (II.36) behaves similar to (II.35) near the horizon. Thus our matching conditions tend to make the normalization integral through $\mathcal{H}_{2}$ a continuous function in $r_{0}$ on the horizon $r_{0}=2 \rho$. Since the integrand of the normalization integral has the interpretation as the "probability density" or "probability current," this means physically that a particle which disappears in the event horizon must reappear in the interior of the horizon. This is in accordance with our physical assumption that there are no other universes or white holes where the particle could disappear into or emerge from. 


\section{SEPARATION OF THE ANGULAR AND TIME DEPENDENCE}

We next study Dirac particles in the external Reissner-Nordström background fields (I.1) and (I.2). Since the external fields are spherically symmetric and time independent, we can separate out the angular and time dependence of the wave functions via spherical harmonics and plane waves, respectively. This is done in a manner similar to the central force problem in Minkowski space (see e.g., Ref. 12).

We start with a compilation of some formulas involving the angular momentum operator $\vec{L}$ $=-i(\vec{x} \times \vec{\nabla})$ (see, e.g., Ref. 13). Its square is

$$
L^{2}=-\Delta_{S^{2}}=L_{+} L_{-}+L_{z}^{2}-L_{z}=L_{-} L_{+}+L_{z}^{2}+L_{z}
$$

with $L_{ \pm}=L_{x} \pm i L_{y}$. The spherical harmonics $Y_{l}^{k}, l=0,1, \ldots, k=-l, \ldots, l$, are simultaneous eigenfunctions of $L^{2}$ and $L_{z}$, namely,

$$
L^{2} Y_{l}^{k}=l(l+1) Y_{l}^{k}, \quad L_{z} Y_{l}^{k}=k Y_{l}^{k} .
$$

They are orthonormal,

$$
\int_{S^{2}} Y_{l}^{k *} Y_{l^{\prime}}^{k^{\prime}}=\delta_{l l^{\prime}} \delta^{k k^{\prime}}
$$

and form a basis of $L^{2}\left(S^{2}\right)$. The operators $L_{ \pm}$serve as "ladder operators," in the sense that

$$
L_{ \pm} Y_{l}^{k}=\sqrt{l(l+1)-k(k \pm 1)} Y_{l}^{k \pm 1} .
$$

In preparation for the four-component Dirac spinors, we consider two-component Pauli spinors. In analogy to (II.2)-(II.4), we denote the "Pauli matrices in polar coordinates" by $\sigma^{r}, \sigma^{\vartheta}$, and $\sigma^{\varphi}$; i.e.,

$$
\begin{gathered}
\sigma^{r}=\sigma^{3} \cos \vartheta+\sigma^{1} \sin \vartheta \cos \varphi+\sigma^{2} \sin \vartheta \sin \varphi, \\
\sigma^{\vartheta}=\frac{1}{r}\left(-\sigma^{3} \sin \vartheta+\sigma^{1} \cos \vartheta \cos \varphi+\sigma^{2} \cos \vartheta \sin \varphi\right), \\
\sigma^{\varphi}=\frac{1}{r \sin \vartheta}\left(-\sigma^{1} \sin \varphi+\sigma^{2} \cos \varphi\right) .
\end{gathered}
$$

We have

$$
\sigma^{\vartheta} \partial_{\vartheta}+\sigma^{\varphi} \partial_{\varphi}=\vec{\sigma} \vec{\nabla}-\sigma^{r} \partial_{r}=\frac{\sigma^{r}}{r}(\vec{\sigma} \vec{x})\left(\vec{\sigma} \vec{\nabla}-\sigma^{r} \partial_{r}\right)=\frac{\sigma^{r}}{r}\left(r \partial_{r}+i \vec{\sigma}(\vec{x} \times \vec{\nabla})-r \partial_{r}\right)=-\frac{\sigma^{r}}{r} \vec{\sigma} \vec{L},
$$

and thus

$$
\vec{\sigma} \vec{L}=-r \sigma^{r}\left(\sigma^{\vartheta} \partial_{\vartheta}+\sigma^{\varphi} \partial_{\varphi}\right) .
$$

For $j=1 / 2,3 / 2, \ldots$ and $k=-j,-j+1, \ldots, j$, we introduce the two-spinors

$$
\begin{gathered}
\chi_{j-1 / 2}^{k}=\sqrt{\frac{j+k}{2 j}} Y_{j-1 / 2}^{k-1 / 2}\left(\begin{array}{l}
1 \\
0
\end{array}\right)+\sqrt{\frac{j-k}{2 j}} Y_{j-1 / 2}^{k+1 / 2}\left(\begin{array}{l}
0 \\
1
\end{array}\right), \\
\chi_{j+1 / 2}^{k}=\sqrt{\frac{j+1-k}{2 j+2}} Y_{j+1 / 2}^{k-1 / 2}\left(\begin{array}{l}
1 \\
0
\end{array}\right)-\sqrt{\frac{j+1+k}{2 j+2}} Y_{j+1 / 2}^{k+1 / 2}\left(\begin{array}{l}
0 \\
1
\end{array}\right) .
\end{gathered}
$$


These spinors form an orthonormal basis of $L^{2}\left(S^{2}\right)^{2}$. They are eigenvectors of the operator $K$ $=\vec{\sigma} \vec{L}+1$. More precisely, (III.1) and (III.2) imply that

$$
\begin{gathered}
K \chi_{j-1 / 2}^{k}=\left(\begin{array}{cc}
L_{z}+1 & L_{-} \\
L_{+} & -L_{z}+1
\end{array}\right) \chi_{j-1 / 2}^{k}=\left(j+\frac{1}{2}\right) \chi_{j-1 / 2}^{k}, \\
K \chi_{j+1 / 2}^{k}=-\left(j+\frac{1}{2}\right) \chi_{j+1 / 2}^{k} .
\end{gathered}
$$

Furthermore, multiplication with $\sigma^{r}$ again gives an eigenvector of $K$; namely,

$$
\begin{aligned}
K \sigma^{r} \chi_{j-1 / 2}^{k} & \stackrel{(\text { III. } 4)}{=}\left(-r \sigma^{r}\left(\sigma^{\vartheta} \partial_{\vartheta}+\sigma^{\varphi} \partial_{\varphi}\right)+1\right) \sigma^{r} \chi_{j-1 / 2}^{k} \\
& =-\sigma^{r} \chi_{j-1 / 2}^{k}-r \sigma^{r}\left(\sigma^{\vartheta} \sigma^{r} \partial_{\vartheta}+\sigma^{\varphi} \sigma_{r} \partial_{\varphi}\right) \chi_{j-1 / 2}^{k} \\
& =-\sigma^{r} \chi_{j-1 / 2}^{k}-\sigma^{r}(\vec{\sigma} \vec{L}) \chi_{j-1 / 2}^{k} \\
& =-\sigma^{r} K \chi_{j-1 / 2}^{k}=-\left(j+\frac{1}{2}\right) \sigma^{r} \chi_{j-1 / 2}^{k} .
\end{aligned}
$$

Taking into account the normalization factors, we obtain the simple formula

$$
\sigma^{r} \chi_{j-1 / 2}^{k}=\chi_{j+1 / 2}^{k} .
$$

Finally, we choose for the Dirac wave functions the two ansatz

$$
\begin{aligned}
& \Psi_{j k \omega}^{+}=e^{-i \omega t} \frac{S^{-1 / 2}}{r}\left(\begin{array}{c}
\chi_{j-1 / 2}^{k} \Phi_{j k \omega 1}^{+}(r) \\
i \chi_{j+1 / 2}^{k} \Phi_{j k \omega 2}^{+}(r)
\end{array}\right), \\
& \Psi_{j k \omega}^{-}=e^{-i \omega t} \frac{S^{-1 / 2}}{r}\left(\begin{array}{c}
\chi_{j+1 / 2}^{k} \Phi_{j k \omega 1}^{-}(r) \\
i \chi_{j-1 / 2}^{k} \Phi_{j k \omega 2}^{-}(r)
\end{array}\right),
\end{aligned}
$$

with the two-spinors $\Phi_{j k \omega}^{+}$and $\Phi_{j k \omega}^{-}$. A general solution of the Dirac equation can be written as a linear combination of these wave functions (this is because one can obtain every combination of spherical harmonics in the four spinor components).

In the regions where the $t$-variable is timelike, we choose the Dirac matrices again in the form (II.1), whereby the function $S$ is now given by

$$
S(r)=\left|1-\frac{2 \rho}{r}+\frac{q^{2}}{r^{2}}\right|^{1 / 2} .
$$

According to (I.4), the formula for the Dirac operator is obtained by inserting the Coulomb potential into (II.5),

$$
G=\gamma^{t}\left(\frac{i}{S} \frac{\partial}{\partial t}-\frac{e}{S} \phi\right)+\gamma^{r}\left(i S \frac{\partial}{\partial r}+\frac{i}{r}(s-1)-\frac{i}{2} S^{\prime}\right)+i \gamma^{\vartheta} \frac{\partial}{\partial \vartheta}+i \gamma^{\varphi} \frac{\partial}{\partial \varphi} .
$$

The identity (III.3) allows us to rewrite the angular derivatives of the Dirac operator in terms of the operator $K$. If we substitute the ansatz (III.8) and (III.9) into the Dirac equation and apply the relations (III.5)-(III.7), we obtain the two-component Dirac equations

$$
S \frac{d}{d r} \Phi_{j k \omega}^{ \pm}=\left[\left(\begin{array}{cc}
0 & -1 \\
1 & 0
\end{array}\right)(\omega-e \phi) \frac{1}{S} \pm\left(\begin{array}{cc}
1 & 0 \\
0 & -1
\end{array}\right) \frac{2 j+1}{2 r}-\left(\begin{array}{ll}
0 & 1 \\
1 & 0
\end{array}\right) m\right] \Phi_{j k \omega}^{ \pm} .
$$

In the regions where the $t$-direction is spacelike, we obtain the generalization of (II.8) for the Dirac operator; namely, 


$$
G=\gamma^{r}\left(\frac{i}{S} \frac{\partial}{\partial t}-\frac{i}{r}-\frac{e}{S} \phi\right)+\gamma^{0}\left(i S \partial_{r}+S \frac{i}{r}+\frac{i}{2} S^{\prime}\right)+i \gamma^{\vartheta} \partial_{\vartheta}+i \gamma^{\varphi} \partial_{\varphi} .
$$

We again choose the ansatz (III.8) and (III.9). This gives the two-component Dirac equations

$$
S \frac{d}{d r} \Phi_{j k \omega}^{ \pm}=\left[\left(\begin{array}{cc}
0 & -1 \\
1 & 0
\end{array}\right)(\omega-e \phi) \frac{1}{S} \pm i\left(\begin{array}{cc}
0 & 1 \\
1 & 0
\end{array}\right) \frac{2 j+1}{2 r}+i\left(\begin{array}{cc}
1 & 0 \\
0 & -1
\end{array}\right) m\right] \Phi_{j k \omega}^{ \pm} .
$$

\section{NONEXTREME REISSNER-NORDSTRÖM BACKGROUND}

In this section, we consider the case $q \neq \rho$, so that the metric coefficient $S(r)$, (III.10), has two zeros

$$
r_{0}=\rho-\sqrt{\rho^{2}-q^{2}} \text { and } \quad r_{1}=\rho+\sqrt{\rho^{2}-q^{2}}
$$

These zeros are transversal, $S^{\prime}\left(r_{j}\right) \neq 0$; in addition, the potential $\phi(r)$ is regular at $r=r_{j}$. Since our matching conditions (II.31) and (II.34) for the Schwarzschild metric only depend on the local behavior of the external field around the horizon, they are also valid for the Reissner-Nordström horizons (for the inner horizon, we must reverse the $r$-direction). We will show in this section that these matching conditions do not admit normalizable, time-periodic solutions of the Dirac equation. More precisely, we will show that for every (nontrivial) solution of the Dirac equation (I.4), the normalization integral outside and away from the horizons,

$$
(\Psi \mid \Psi)_{\infty}^{t}:=\int_{\mathbb{R}^{3} B_{2 r_{1}}} \bar{\Psi} \gamma^{t} \Psi S^{-1} d^{3} x
$$

is infinite for some $t$. Notice that for a normalized wave function, the integral (IV.1) gives the probability that the particle lies outside the ball of radius $2 r_{1}$, which must be smaller than one. Thus, if (IV.1) is inifinite, the wave function cannot be normalized.

Suppose that we have a periodic solution (I.3) of the Dirac equation with period T. Expanding the periodic function $e^{i \Omega t} \Psi(t, r, \vartheta, \varphi)$ in a Fourier series gives the representation of $\Psi$ (as the Bloch wave)

$$
\Psi(t, r, \vartheta, \varphi)=e^{-i \Omega t} \sum_{n \in Z} \Psi_{n}(r, \vartheta, \varphi) e^{-2 \pi i n t / T}
$$

Decomposing the functions $\Psi_{n}$ in the basis (III.8) and (III.9) and substituting into (IV.2) gives

$$
\Psi(t, r, \vartheta, \varphi)=\sum_{n, j, k, s} \Psi_{j k \omega(n)}^{s}(t, r, \vartheta, \varphi)
$$

where the index $s= \pm$, and where $\omega$ is related to $n$ by

$$
\omega(n)=\Omega+\frac{2 \pi n}{T}
$$

Using the orthonormality of the two-spinors $\chi_{j \pm 1 / 2}^{k}$, the normalization integral takes the form

$$
(\Psi \mid \Psi)_{\infty}^{t}=\int_{\mathbb{R}^{3} \backslash B_{2 r_{1} n, n^{\prime}}} \sum_{j, k, s} \bar{\Psi}_{j k \omega(n)}^{s} \gamma^{t} \Psi_{j k \omega\left(n^{\prime}\right)}^{s} S^{-1} d^{3} x
$$

The integrand has an oscillating time dependence of the form $\exp \left(i\left(\omega(n)-\omega\left(n^{\prime}\right)\right) t\right)$. In order to eliminate the oscillations, we take the average over one period $(0, T)$, giving 


$$
\frac{1}{T} \int_{0}^{T}(\Psi \mid \Psi)_{\infty}^{t} d t=\sum_{n, j, k, s}\left(\Psi_{j k \omega(n)}^{s} \mid \Psi_{j k \omega(n)}^{s}\right)_{\infty}
$$

For a normalizable wave function $\Psi$, this expression is finite. Since the scalar product $(. \mid .)_{\infty}$ is (semi-)positive definite, we conclude that all the summands must be finite; thus

$$
\left(\Psi_{j k \omega(n)}^{s} \mid \Psi_{j k \omega(n)}^{s}\right)<\infty
$$

for all $s= \pm, j, k, n$.

This inequality allows us to turn our attention to the individual wave functions $\Psi_{j k \omega}^{s}$. As a first step we show that the wave functions $\Phi^{ \pm}$in the ansatz (III.8) and (III.9) are not zero on the horizon.

Lemma IV.1: The function $\left|\Phi_{j k \omega}^{ \pm}(r)\right|^{2}$ has finite boundary values on the horizon. If it is zero on a horizon $r=r_{0}$ or $r=r_{1}$, then $\Phi_{j k \omega}^{+}$vanishes identically.

Proof: For ease in notation, we omit the indices $j, k$, and $\omega$. For a given $\delta, 0<\delta<r_{0}$, the $t$-direction is timelike in the regions $\left(\delta, r_{0}\right)$ and $\left(r_{1}, \infty\right)$. In these regions, the Dirac equations (III.12) give

$$
\begin{aligned}
S \frac{d}{d r}\left|\Phi^{ \pm}\right|^{2}(r) & =\left\langle S \frac{d}{d r} \Phi^{ \pm}, \Phi^{ \pm}\right\rangle+\left\langle\Phi^{ \pm}, S \frac{d}{d r} \Phi^{ \pm}\right\rangle \\
& = \pm \frac{2 j+1}{r}\left(\left|\Phi_{1}^{ \pm}\right|^{2}-\left|\Phi_{2}^{ \pm}\right|^{2}\right)-4 m \operatorname{Re}\left(\left(\Phi_{1}^{ \pm}\right)^{*} \Phi_{2}^{ \pm}\right),
\end{aligned}
$$

and thus

$$
-c\left|\Phi^{ \pm}\right|^{2} \leqslant S \frac{d}{d r}\left|\Phi^{ \pm}\right|^{2} \leqslant c\left|\Phi^{ \pm}\right|^{2}
$$

with $c=2 m+(2 j+1) / \delta$. Dividing by $\left|\Phi^{ \pm}\right|^{2}$ and integrating yields, for $\delta<r<r^{\prime}<r_{0}$, or $r_{1}<r$ $<r^{\prime}$, the inequality

$$
-c \int_{r}^{r^{\prime}} S^{-1} \leqslant\left.\log \left|\Phi^{ \pm}\right|^{2}\right|_{r} ^{r^{\prime}} \leqslant c \int_{r}^{r^{\prime}} S^{-1} .
$$

In the region $r_{0}<r<r_{1}$, the Dirac equations (III.14) give similarly

$$
S \frac{d}{d r}\left|\Phi^{ \pm}\right|^{2}(r)=\left\langle S \frac{d}{d r} \Phi^{ \pm}, \Phi^{ \pm}\right\rangle+\left\langle\Phi^{ \pm}, S \frac{d}{d r} \Phi^{ \pm}\right\rangle=0,
$$

since the square bracket in (III.14) is an anti-Hermitian matrix. Thus $\left|\Phi^{ \pm}\right|^{2}$ is constant in this region, and, so, (IV.5) also (trivially) holds for $r_{0}<r<r^{\prime}<r_{1}$.

Notice that $S^{-1}$ is integrable on the event horizons. Therefore, the inequality (IV.5) implies that the left- and right-sided boundary values of $\left|\Phi^{ \pm}\right|^{2}$ on the horizon are finite, and are nonzero unless $\Phi^{ \pm}$vanishes identically in the corresponding region $\left(\delta, r_{0}\right),\left(r_{0}, r_{1}\right)$, or $\left(r_{1}, \infty\right)$.

Next we consider the matching condition (II.34). If we substitute the ansatz (III.8) and (III.9), we get for $\Phi^{ \pm}$the conditions

$$
\Phi^{ \pm}\left(r_{j}+\varepsilon\right)-\Phi^{ \pm}\left(r_{j}-\varepsilon\right)=o\left(1+\left|\Phi^{ \pm}\left(r_{j}+\varepsilon\right)\right|\right) \quad \text { at } \varepsilon \rightarrow 0, j=0,1 .
$$

Since we have already shown that $\left|\Phi^{ \pm}(r)\right|^{2}$ has two-sided limits as $r=r_{j}$, this last equality shows that the left- and right-sided boundary values of $\left|\Phi^{ \pm}\right|^{2}$ must coincide,

$$
\lim _{0<\varepsilon \rightarrow 0}\left|\Phi^{ \pm}\left(r_{j}+\varepsilon\right)\right|^{2}=\lim _{0<\varepsilon \rightarrow 0}\left|\Phi^{ \pm}\left(r_{j}-\varepsilon\right)\right|^{2}, \quad j=0,1 .
$$


We conclude that the wave function can only be zero on one of the horizons if it vanishes in the whole interval $(\delta, \infty)$. Taking the limit $\delta \rightarrow 0$ gives the result.

We point out that this lemma does not imply that the wave function $\Phi$ is continuous on the horizon. In general, $\Phi(r)$ will oscillate faster and faster as $r$ approaches a horizon. Nevertheless, its absolute value $|\Phi|$ tends to a finite value in this limit.

The next step is to use current conservation for analyzing the decay of $\Psi_{j k \omega(n)}^{s}$ at infinity.

Theorem IV.2 (radial flux argument): Either $\Psi_{j k \omega}^{s}$ vanishes identically, or the normalization condition (IV.4) is violated.

Proof: To simplify the notation, we again omit the indices $s, j, k$, and $\omega$. Assume that $\Psi$ is not identically zero. For $r_{1}<r<R$ and $T>0$, let $V=(0, T) \times\left(B_{2 R} \backslash B_{2 r}\right)$ be an annulus outside the horizon. As a consequence of the current conservation, the flux integral over the boundary of $V$ is zero, thus

$$
\begin{aligned}
0= & \int_{V}+\nabla_{j}\left(\bar{\Psi} G^{j} \Psi\right) \sqrt{|g|} d^{4} x \\
= & \int_{0}^{T} d t r^{2} S(r) \int_{S^{2}}\left(\bar{\Psi} \gamma^{r} \Psi\right)(t, r)-\int_{0}^{T} d t R^{2} S(R) \int_{S^{2}}\left(\bar{\Psi} \gamma^{r} \Psi\right)(t, R) \\
& -\left.\int_{2 r}^{2 R} d s s^{2} S^{-1}(s) \int_{S^{2}}\left(\bar{\Psi} \gamma^{t} \Psi\right)(t, r)\right|_{t=0} ^{t=T}
\end{aligned}
$$

where $\int_{S^{2}}$ denotes the integral over the angular variables. Since the integrand is static, the last integral vanishes, and we obtain that the radial flux is independent of the radius,

$$
r^{2} S(r) \int_{S^{2}}\left(\bar{\Psi} \gamma^{r} \Psi\right)(r)=R^{2} S(R) \int_{S^{2}}\left(\bar{\Psi} \gamma^{r} \Psi\right)(R)
$$

We want to show that the radial flux is not zero. For this, we first substitute the ansatz (III.8) and (III.9) into the right side of (IV.6) and get

$$
r^{2} S(r) \int_{S^{2}}\left(\bar{\Psi} \gamma^{r} \Psi\right)(r)=\int_{S^{2}} \Phi^{*}(r)\left(\begin{array}{cc}
0 & i \\
-i & 0
\end{array}\right) \Phi(r) .
$$

According to Lemma IV.1, $|\Phi|$ has finite, nonzero boundary values on the horizon $r_{1}$. Expressed in $\Phi$, the matching condition (II.31) gives

$$
\lim _{r_{1}<r \rightarrow r_{1}}\left(\begin{array}{cc}
1 & i \\
i & -1
\end{array}\right) \Phi=0 .
$$

Using this equation, we take the limit $r \rightarrow r_{1}$ in (IV.7),

$$
\begin{aligned}
\lim _{r_{1}<r \rightarrow r_{1}} r^{2} S(r) \int_{S^{2}}\left(\bar{\Psi} \gamma^{r} \Psi\right)(r) & =\lim _{r_{1}<r \rightarrow r_{1}} \int_{S^{2}}\left[\Phi *\left(\begin{array}{cc}
1 & i \\
-i & 1
\end{array}\right) \Phi-|\Phi|^{2}\right] \\
& =\lim _{r_{1}<r \rightarrow r_{1}} \int_{S^{2}}\left[\Phi *\left(\begin{array}{cc}
1 & 0 \\
0 & -1
\end{array}\right)\left(\begin{array}{cc}
1 & i \\
i & -1
\end{array}\right) \Phi-|\Phi|^{2}\right] \\
& =-\lim _{r_{1}<r \rightarrow r_{1}} \int_{S^{2}}|\Phi|^{2} \neq 0,
\end{aligned}
$$

where we used Lemma IV.1 in the last inequality 
Now we consider the radial flux for large $R$. Since the flux is nonzero and independent of $R$, we have

$$
0<\lim _{R \rightarrow \infty}\left|R^{2} S(R) \int_{S^{2}}\left(\bar{\Psi} \gamma^{r} \Psi\right)(R)\right|
$$

Using the positivity of the form $\bar{\Psi} \gamma^{t} \Psi$ and the fact that the Reissner-Nordström metric is asymptotically Minkowskian, we get (using the Cauchy-Schwarz inequality) the estimate

$$
\begin{aligned}
& 0<\lim _{R \rightarrow \infty}\left|R^{2} S(R) \int_{S^{2}}\left(\bar{\Psi} \gamma^{r} \Psi\right)(R)\right| \leqslant \lim _{R \rightarrow \infty}\left|R^{2} S(R) \int_{S^{2}}\left(\bar{\Psi} \gamma^{t} \Psi\right)(R)\right| \\
& \quad=\lim _{R \rightarrow \infty}\left|R^{2} S^{-1}(R) \int_{S^{2}}\left(\bar{\Psi} \gamma^{t} \Psi\right)(R)\right|
\end{aligned}
$$

We have shown that the integrand of our normalization integral

$$
(\Psi \mid \Psi)_{\infty}=\int_{2 r_{1}}^{\infty} d R R^{2} S^{-1}(R) \int_{S^{2}}\left(\Psi \gamma^{t} \Psi\right)(R)
$$

converges to a positive number. Thus the normalization integral must be infinite.

This theorem shows that the wave functions $\Psi_{j k \omega}^{s}$ in the decomposition (IV.2) and (IV.3) must all be identically zero. Thus there are no normalizable solutions of the Dirac equation; this proves Theorem I.1.

Remark IV.3: We point out that the radial flux argument is based only on our matching conditions for the wave functions and on the Dirac current conservation. Therefore, it can immediately be applied to more general static, spherically symmetric background fields. This generalization may, for example, be relevant if the coupling of the gravitational and electric field to matter or other force fields is taken into account. Although the exact formulas of the Reissner-Nordström solution will then no longer be valid, the qualitative behavior of the fields on the horizons may still be the same. To give an example of the possible generalizations, we state the following theorem, which can be proved with very similar methods: Let $g_{i j}$ be a static, radially symmetric background metric,

$$
d s^{2}=g_{i j} d x^{i} d x^{j}=\frac{1}{T^{2}(r)} d t^{2}-\frac{1}{A(r)} d r^{2}-r^{2}\left(d \vartheta^{2}+\sin ^{2} \vartheta d \varphi^{2}\right),
$$

whereby the metric coefficient $A(r)$ has $N$ zeros at $r=r_{1}, \ldots, r_{N}, 0<r_{0}<\cdots<r_{N}$. Assume the following conditions hold:

(1) The zeros of A are all transversal,

$$
A^{\prime}\left(r_{j}\right) \neq \quad \text { for } j=1, \ldots, N \text {. }
$$

(2) The determinant of the metric is regular except at the origin,

$$
T^{-2}(r) A^{-1}(r) \in C^{\infty}(0, \infty) .
$$

Furthermore, assume there is a spherically symmetric electric field $\phi(r)$ which is regular except at the origin, $\phi \in C^{\infty}(0, \infty)$. Then there are no normalizable, time-periodic solutions of the Dirac equation with these background fields. 


\section{EXTREME REISSNER-NORDSTRÖM BACKGROUND}

We now consider the case $q=\rho$ of an extreme Reissner-Nordström background field, i.e.,

$$
S=\frac{r-\rho}{r}
$$

The metric coefficient $S$ now has only one zero at $r=\rho$; the $t$-direction is timelike both inside and outside the horizon. This situation can be thought of as the limiting case that the two horizons $r_{0}$ and $r_{1}$ considered in the previous section come arbitrarily close. Unfortunately, the arguments for the nonexistence proof do not carry over in this limit, so we must rely on a different method.

Since the $t$-direction is always timelike, the $t$-component of the current $\bar{\Psi} G^{t} \Psi$ is positive and has the usual interpretation as probability density. Therefore, the normalization integral

$$
(\Psi \mid \Psi)^{t}=\int_{\mathbb{R}^{3}} \bar{\Psi} \gamma^{t} \Psi S^{-1} d^{3} x
$$

causes no conceptual difficulties.

Suppose that we had a normalizable, periodic solution (I.3) of the Dirac equation with period T. Again, using the representation as the Bloch wave (IV.2) and averaging over one period gives

$$
\infty>\frac{1}{T} \int_{0}^{T}(\Psi \mid \Psi)^{t} d t=\sum_{n, j, k, s}\left(\Psi_{j k \omega(n)}^{s} \mid \Psi_{j k \omega(n)}^{s}\right) .
$$

Substituting the ansatz (III.8) and (III.9) yields

$$
\frac{1}{T} \int_{0}^{T}(\Psi \mid \Psi)^{t} d t=\int_{0}^{\infty} d r S^{-2}(r) \sum_{n, j, k, s}\left|\Phi_{j k \omega(n)}^{s}\right|^{2} .
$$

Using the positivity of the summands, we obtain the conditions

$$
\int_{0}^{\infty} d r S^{-2}(r)\left|\Phi_{j k \omega(n)}^{s}\right|^{2}<\infty
$$

for all $s, j, k$, and $n$.

We will now study the individual functions $\Phi_{j k \omega}^{s}$ for $r>\rho$. To simplify the notation, we again omit the indices $j, k$, and $\omega$. Our first task is to consider under which conditions on the parameters $\omega, j$, and $m$ the normalization integral (V.1) can be finite near $r=\rho$. We first discuss the situation qualitatively: Since $S^{-2}(r)=r^{2} /(r-\rho)^{2}$ has a nonintegrable singularity on the horizon, the normalization integral will only be finite if $\Phi^{s}$ becomes small near $r=\rho$. For generic parameter values, the dominant term in the Dirac equation (III.12) near $r=\rho$ is the first summand, i.e.,

$$
\frac{d}{d r} \Phi^{ \pm} \approx \frac{\omega-e \phi}{S^{2}}\left(\begin{array}{cc}
0 & -1 \\
1 & 0
\end{array}\right) \Phi^{ \pm} .
$$

Since, in this limiting case, the eigenvalues of the matrix on the right are purely imaginary, the Dirac equation describes fast oscillations of the wave function. The eigenvalues of the second and third summands in (III.12) are real; they describe an exponential increase or decay of $\Phi$. If the oscillating term is dominant, we expect that $\Phi$ will not go to zero in the limit $r \rightarrow \rho$. In the following lemma, these ideas are made mathematically precise in a slightly more general setting.

Lemma V.1: Let $\Phi(x), x>0$, be a nontrivial solution of the $O D E$

$$
\Phi^{\prime}(x)=\left[a(x)\left(\begin{array}{cc}
0 & -1 \\
1 & 0
\end{array}\right)+b(x)\left(\begin{array}{cc}
1 & 0 \\
0 & -1
\end{array}\right)+c(x)\left(\begin{array}{ll}
0 & 1 \\
1 & 0
\end{array}\right)\right] \Phi(x)
$$


with smooth, real functions $a, b, c \in C^{\infty}(0, \infty)$ and $a \neq 0$. If, near the origin, the quotients $b / a$ and cla are monotone and

$$
b(x)^{2}+c(x)^{2}<a(x)^{2},
$$

then $|\Phi|^{2}(x)$ is bounded from above and from below near $x=0$,

$$
0<\lim _{0<x \rightarrow 0} \inf |\Phi(x)|^{2} \leqslant \lim _{0<x \rightarrow 0} \sup |\Phi(x)|^{2}<\infty .
$$

Proof: Let $(0, \varepsilon)$ be an interval where the functions $b / a$ and $c / a$ are monotone and where (V.3) holds. Assume that $\Phi$ is a nontrivial solution of (V.2). According to the uniqueness theorem for the solutions of ODEs, $\Phi(x)$ is nonzero for all $0<x<\infty$. Now consider the functional

$$
F(x)=\langle\Phi(x), A(x) \Phi(x)\rangle \quad \text { with } A(x)=\left(\begin{array}{cc}
1+b / a & -c / a \\
-c / a & 1-b / a
\end{array}\right) .
$$

According to (V.3), the matrix $A$ is close to the identity; i.e., there is a constant $c<1$ with

$$
|1-A(x)|<c \quad \text { for all } x \text { with } 0<x<\varepsilon .
$$

Thus the functional $F$ is uniformly bounded in $|\Phi|^{2}$ on $(0, \varepsilon)$,

$$
\frac{1}{C}|\Phi(x)|^{2} \leqslant F(x) \leqslant C|\Phi(x)|^{2}
$$

for some $C>0$. Using the special form of $A$ and of the differential equation (V.2), the derivative of $F$ takes the simple form

$$
F^{\prime}(x)=\left\langle\Phi^{\prime}, A \Phi\right\rangle+\left\langle\Phi, A \Phi^{\prime}\right\rangle+\left\langle\Phi, A^{\prime} \Phi\right\rangle=\left\langle\Phi, A^{\prime} \Phi\right\rangle .
$$

The sup-norm of the matrix $A^{\prime}$ is bounded by

$$
\left\{A^{\prime}\right\} \leqslant\left|\left(\frac{b}{a}\right)^{\prime}\right|+\left|\left(\frac{c}{a}\right)^{\prime}\right| .
$$

Putting together (V.4)-(V.6), we get the bounds

$$
-C\left(\left|\left(\frac{b}{a}\right)^{\prime}\right|+\left|\left(\frac{c}{a}\right)^{\prime}\right|\right) F(x) \leqslant F^{\prime}(x) \leqslant C\left(\left|\left(\frac{b}{a}\right)^{\prime}\right|+\left|\left(\frac{c}{a}\right)^{\prime}\right|\right) F(x) .
$$

Now we divide by $F(x)$ and integrate. Since $b / a$ and $c / a$ are monotone, we can just integrate inside the absolute values,

$$
-\left.C\left(\left|\frac{b}{a}\right|+\left|\frac{c}{a}\right|\right)\right|_{x} ^{y} \leqslant\left.\log F\right|_{x} ^{y} \leqslant\left. C\left(\left|\frac{b}{a}\right|+\left|\frac{c}{a}\right|\right)\right|_{x} ^{y} .
$$

Since the extreme left and right sides of this inequality converge in the limit $x \rightarrow 0$, we conclude that $\log F(x)$ is bounded from above and below near the origin. After exponentiating and substituting (V.4), the result follows. unless

Applied to (III.12), this lemma says that $\left|\Phi^{ \pm}(r)\right|^{2}$ is bounded away from zero near $r=\rho$

$$
\omega-e \phi(\rho)=0 .
$$

Thus we can turn our attention to this special case. 
If we substitute the condition (V.8) into (III.12), the Dirac equation simplifies to

$$
\left|1-\frac{\rho}{r}\right| \frac{d}{d r} \Phi^{ \pm}(r)=\left[\left(\begin{array}{cc}
0 & -1 \\
1 & 0
\end{array}\right) e \pm\left(\begin{array}{cc}
1 & 0 \\
0 & -1
\end{array}\right) \frac{2 j+1}{2 r}-\left(\begin{array}{ll}
0 & 1 \\
1 & 0
\end{array}\right) m\right] \Phi^{ \pm} .
$$

We want to study how the solutions of this equation behave for small $r-\rho>0$. For this, we rewrite the equation in the new variable

$$
u(r)=-r-\rho \ln (r-\rho)
$$

which gives

$$
\frac{d}{d u} \Phi^{ \pm}(u)=\left[-\left(\begin{array}{cc}
0 & -1 \\
1 & 0
\end{array}\right) e \mp\left(\begin{array}{cc}
1 & 0 \\
0 & -1
\end{array}\right) \frac{2 j+1}{2 r}+\left(\begin{array}{ll}
0 & 1 \\
1 & 0
\end{array}\right) m\right] \Phi^{ \pm}
$$

The region near $r=\rho$ corresponds to large values of $u$. The matrix in the bracket in (V.10) depends smoothly on $u$ and converges in the limit $u \rightarrow \infty$ to a finite limit, in view of the definition of $u$ given above. According to the stable manifold theorem (Ref. 14, Thm. 4.1), the solutions of (V.10) which are not bounded away from zero for large $u$ tend exponentially to zero. After transforming back to the variable $r$, this justifies the power ansatz

$$
\Phi_{1}^{ \pm}(r)=\Phi_{10}^{ \pm}(r-\rho)^{s}+o\left((r-\rho)^{s}\right), \quad \Phi_{2}^{ \pm}(r)=\Phi_{20}^{ \pm}(r-\rho)^{s}+o\left((r-\rho)^{s}\right)
$$

with constants $\Phi_{10}^{ \pm}, \Phi_{20}^{ \pm}$and a parameter $s>0$. Substituting into (V.9) yields the system of linear equations

$$
\begin{aligned}
& \left(s \mp\left(j+\frac{1}{2}\right)\right) \Phi_{10}^{ \pm}=-\rho(m+e) \Phi_{20}^{ \pm}, \\
& \left(s \pm\left(j+\frac{1}{2}\right)\right) \Phi_{20}^{ \pm}=-\rho(m-e) \Phi_{10}^{ \pm},
\end{aligned}
$$

which can be solved for $\Phi_{10}^{ \pm}$and $\Phi_{20}^{ \pm}$. In this way, we have found a consistent ansatz for the spinors near $r=\rho$. However, the corresponding solutions of the Dirac equation are all not normalizable, as the following theorem shows.

Theorem V.2: Every nontrivial solution $\Phi^{ \pm}(r), r>\rho$, of the Dirac equation (V.9) with the boundary conditions (V.11) violates the normalization condition (V.1).

Proof: Let $\Phi^{ \pm}$be a nontrivial solution of the Dirac equation. Since the Dirac equation has real coefficients, we can assume that $\Phi^{ \pm}$are real. In the new variable $u=r^{-1}$, the Dirac equation (V.9) takes the form

$$
|1-\rho u| \frac{d}{d u} \Phi^{ \pm}(u)=\left[-\frac{e}{u^{2}}\left(\begin{array}{cc}
0 & -1 \\
1 & 0
\end{array}\right) \mp \frac{2 j+1}{2 u}\left(\begin{array}{cc}
1 & 0 \\
0 & -1
\end{array}\right)+\frac{m}{u^{2}}\left(\begin{array}{cc}
0 & 1 \\
1 & 0
\end{array}\right)\right] \Phi^{ \pm}
$$

If $e>m$, Lemma V.1 yields that $\left|\Phi^{ \pm}(u)\right|^{2}$ is bounded from above and below near $u=0$. Thus $\left|\Phi^{ \pm}(r)\right|^{2}$ does not decay at infinity, and the normalization integral (V.1) will diverge. We conclude that we must only consider the case $m \geqslant e$.

In the case $m=e$, the system (V.12) and (V.13) yields that either $\Phi_{10}^{ \pm}$or $\Phi_{20}^{ \pm}$is zero. Furthermore, the Dirac equation (V.9) shows that either $\Phi_{1}^{ \pm}$or $\Phi_{2}^{ \pm}$vanishes identically. Since $\Phi^{ \pm}(r)$ has no zeros for finite $r$ (otherwise, the uniqueness of the solution yields that $\Phi^{ \pm}$vanishes identically), we can assume that the vector $\Phi^{ \pm}(r)$ will lie in the fourth quadrant,

$$
\Phi^{ \pm}(r) \in\{(x, y) \mid x \geqslant 0, y \leqslant 0\}
$$

for all $r$.

Next we want to show that (V.14) also holds in the case $m>e$. In this case, from (V.12) and (V.13), we can assume that $\Phi_{10}^{ \pm}$is positive, whereas $\Phi_{20}^{ \pm}$is negative. Thus (V.14) holds for small 


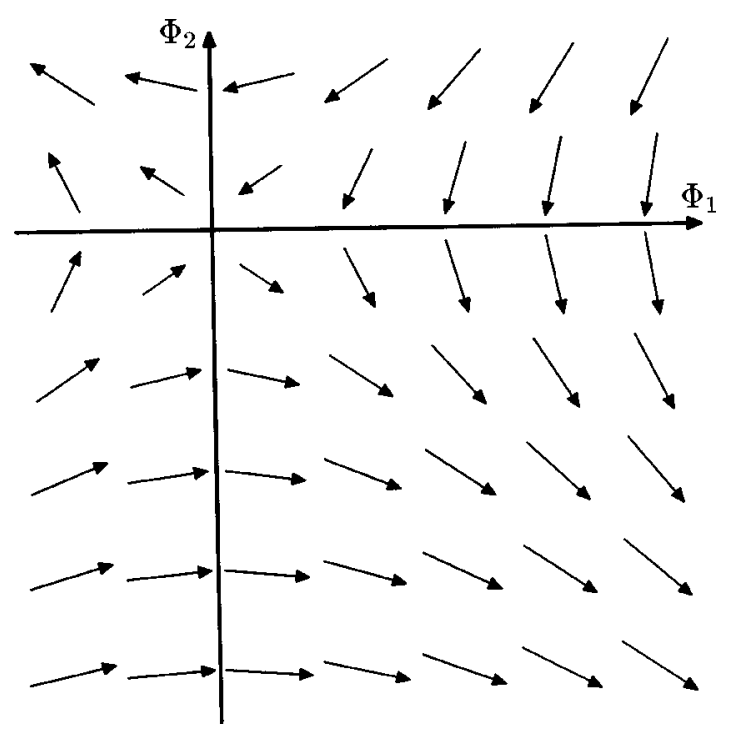

FIG. 2. Flow of $\Phi^{ \pm}$for large $r$, schematic.

$r-\rho>0$. In order to show that the fourth quadrant is an invariant region for $\Phi^{ \pm}$, first notice that $\Phi^{ \pm}(r)$ cannot become zero for a finite value of $r$. Thus, if $\Phi^{ \pm}(r)$ leaves the quadrant for some $r$, we have either

$$
\Phi_{1}^{ \pm}(r)=0, \quad\left(\Phi_{1}^{ \pm}\right)^{\prime}(r) \leqslant 0, \quad \text { and } \quad \Phi_{2}^{ \pm}(r)<0
$$

or

$$
\Phi_{1}^{ \pm}(r)>0, \quad \Phi_{2}^{ \pm}(r)=0, \quad \text { and } \quad\left(\Phi_{2}^{ \pm}\right)^{\prime}(r) \geqslant 0 .
$$

However, the Dirac equation gives in the first case that $\left(\Phi_{1}^{ \pm}\right)^{\prime}>0$ and in the second case that $\left(\Phi_{2}^{ \pm}\right)^{\prime}(r)<0$, which is a contradiction.

We conclude that $\Phi^{ \pm}(r)$ lies for all $r$ in the fourth quadrant. Figure 2 shows the flow of Eq. (V.9) for large $r$. From this one sees immediately that the origin is repelling, so that $\left|\Phi^{ \pm}\right|^{2}$ will be bounded away from zero for large $r$.

It follows that our periodic solution $\Psi$ must vanish identically outside the horizon. This proves Theorem I.2.

We point out that in contrast to the situation in Sec. IV, we do not make any statement on the behavior of the wave function for $r<\rho$. Indeed, it appears that the extreme Reissner-Nordström background does admit periodic solutions for $r<\rho$; these can be constructed by taking the boundary conditions (V.11) on the horizon and solving the Dirac equation backwards in $r$.

\section{ACKNOWLEDGMENTS}

JS was supported in part by NSF Grant No. DMS-G-9501128, and STY was supported in part by NSF Grant No. 33-585-7510-2-30.

\section{APPENDIX: JUSTIFICATION OF TIME PERIODICITY INSIDE THE HORIZON}

Throughout this paper, we have considered a Dirac wave function (I.3) which is time periodic both inside and outside the event horizon. Since an outside observer has no knowledge about the physical situation in the interior of the event horizon, the assumption of time periodicity inside the horizon might not seem physically reasonable. In this short appendix, we clarify why time periodicity inside the horizon is natural to assume. Namely, we show that every solution $\Psi(t, r, \vartheta, \varphi)$ 
of the Dirac equation which is time periodic outside the event horizon and (locally uniformly) bounded in $t$, gives rise to a solution $\Psi$ of the Dirac equation, which coincides with $\Psi$ outside the horizon and is also time periodic inside. Using this argument, the results of this paper could be immediately generalized to Dirac wave functions which are only time periodic outside the event horizon.

Let $\Psi(t, r, \vartheta, \varphi)$ be a solution of the Dirac equation which is time periodic outside the event horizon,

$$
\Psi(t+T, r, \vartheta, \varphi)=e^{-i \Omega T} \Psi(t, r, \vartheta, \varphi) \quad \text { for } r>r_{1},
$$

and locally uniformly bounded in $t$,

$$
|\Psi(t, r, \vartheta, \varphi)| \leqslant F(r) \quad \text { with } F \in C^{0}\left(\left(0, r_{0}\right) \cup\left(r_{0}, r_{1}\right)\right)
$$

( $r_{0}$ and $r_{1}$ again denote the Cauchy and event horizons, respectively). We consider for $N \geqslant 1$ the functions

$$
\widetilde{\Psi}_{N}(t, r, \vartheta, \varphi)=\frac{1}{2 N+1} \sum_{n=-N}^{N} \Psi(t+n T, r, \vartheta, \varphi)
$$

Since our Dirac operator is static, the functions $\widetilde{\Psi}_{N}$ satisfy the Dirac equation. Time-periodicity (A1) implies that $\widetilde{\Psi}_{N}$ and $\Psi$ coincide outside the event horizon. Inside the event horizon, one can use the bound (A2) to show that the $\widetilde{\Psi}_{N}$ form a Cauchy sequence. Thus we can take the limit $N \rightarrow \infty$; we set $\widetilde{\Psi}=\lim _{N \rightarrow \infty} \widetilde{\Psi}_{N}$. Again using (A2), we conclude that the function $\widetilde{\Psi}$ is time periodic,

$$
\widetilde{\Psi}(t+T, r, \vartheta, \varphi)-\widetilde{\Psi}(t, r, \vartheta, \varphi)=\lim _{N \rightarrow \infty} \frac{1}{2 N+1}(\Psi(t+(N+1) T, r, \vartheta, \varphi)-\Psi(t-N T, r, \vartheta, \varphi))=0,
$$

and satisfies the Dirac equation,

$$
(G-m) \widetilde{\Psi}=\lim _{N \rightarrow \infty}(G-m) \widetilde{\Psi}_{N}=0
$$

${ }^{1}$ D. Christodoulou, "The formation of black holes and singularities in spherically symmetric gravitational collapse," Commun. Pure Appl. Math. 44, 339-373 (1991).

${ }^{2}$ M. W. Choptuik, "Universality and scaling in the gravitational collapse of a scalar field," Phys. Rev. Lett. 70, 9-12 (1993).

${ }^{3}$ J.-P. Nicolas, "Scattering of linear Dirac fields by a spherically symmetric black hole," Ann. Inst. H. Poincaré Physique theorique 62, 145-179 (1995).

${ }^{4}$ J.-P. Nicolas, “Opérateur de diffusion pour le système de Dirac en métrique de Schwarzschild," C. R. Acad. Sci., Ser. I: Math. 318, 729-734 (1994).

${ }^{5}$ S. W. Hawking, "Particle creation by black holes," Commun. Math. Phys. 43, 199-220 (1975).

${ }^{6}$ R. Wald, General Relativity (Univ. of Chicago, Chicago, 1984).

${ }^{7}$ J. Smoller and A. Wasserman, "Uniqueness of the extreme Reissner-Nordström solution in SU(2) Einstein-Yang-Mills theory for spherically symmetric space-time,"' Phys. Rev. D 52, 5812-5815 (1995).

${ }^{8}$ F. Finster, "Local U(2, 2) symmetry in relativistic quantum mechanics," J. Math. Phys. 39, 6276-6290 (1998); hep-th/9703083.

${ }^{9}$ F. Finster, J. Smoller, and S.-T. Yau, “Particlelike solutions of the Einstein-Dirac equations," Phys. Rev. D 59, 104020 (1999); gr-qc/9801079.

${ }^{10}$ F. Finster, J. Smoller, and S.-T. Yau, "Particlelike solutions of the Einstein-Dirac-Maxwell equations," Phys. Lett. A 259, 431-436 (1999); gr-qc/9802012.

${ }^{11}$ R. Adler, M. Bazin, and M. Schiffer, Introduction to General Relativity, 2nd ed. (McGraw-Hill, New York, 1975).

${ }^{12}$ J. J. Sakurai, Advanced Quantum Mechanics (Addison-Wesley, Reading, MA, 1967).

${ }^{13}$ L. D. Landau and E. M. Lifshitz, Quantum Mechanics (Pergamon, Oxford, 1977).

${ }^{14}$ E. Coddington and N. Levinson, Theory of Ordinary Differential Equations (McGraw-Hill, New York, 1955). 